PROCEEDINGS OF THE

AMERICAN MATHEMATICAL SOCIETY

Volume 138, Number 8, August 2010, Pages 2863-2872

S 0002-9939(10)10284-6

Article electronically published on March 23, 2010

\title{
POINT CONFIGURATIONS THAT ARE ASYMMETRIC YET BALANCED
}

\author{
HENRY COHN, NOAM D. ELKIES, ABHINAV KUMAR, AND ACHILL SCHÜRMANN
}

(Communicated by Jim Haglund)

\begin{abstract}
A configuration of particles confined to a sphere is balanced if it is in equilibrium under all force laws (that act between pairs of points with strength given by a fixed function of distance). It is straightforward to show that every sufficiently symmetrical configuration is balanced, but the converse is far from obvious. In 1957 Leech completely classified the balanced configurations in $\mathbb{R}^{3}$, and his classification is equivalent to the converse for $\mathbb{R}^{3}$. In this paper we disprove the converse in high dimensions. We construct several counterexamples, including one with trivial symmetry group.
\end{abstract}

\section{INTRODUCTION}

A finite configuration of points on the unit sphere $S^{n-1}$ in $\mathbb{R}^{n}$ is balanced if it in equilibrium (possibly unstable) under all pairwise forces depending only on distance, assuming the points are confined to the surface of the sphere. In other words, the net forces acting on the points are all orthogonal to the sphere. As is usual in physics, any two distinct particles exert forces on each other, directed oppositely and with magnitude equal to some fixed function of the Euclidean distance between them. The net force on each point is the sum of the contributions from the other points.

For example, the vertices of any regular polyhedron are balanced. On the other hand, most configurations are not balanced. Even if some points are in equilibrium under one force law, there is no reason to expect that they will be in equilibrium under every force law, and usually they will not be. The balanced configurations are quite remarkable.

The condition of being balanced was defined by Leech in [L. It arises in the search for energy-minimizing point configurations on spheres. Given a potential function, typically an inverse-power law, how should we arrange some particles to minimize the total potential energy? This problem originated in Thomson's model of the atom in [T, p. 255]. Of course, that model was superseded by quantum

Received by the editors March 30, 2009.

2010 Mathematics Subject Classification. Primary 52B15; Secondary 05B40, 52C17, 82B05.

The first, second, and third authors thank the Aspen Center for Physics for its hospitality and support. The first, third, and fourth authors thank the Hausdorff Research Institute for Mathematics. The third and fourth authors thank Microsoft Research. The third author was supported in part by a Clay Liftoff Fellowship and by National Science Foundation grant DMS0757765. The second author was supported in part by NSF grant DMS-0501029, and the fourth author was supported in part by Deutsche Forschungsgemeinschaft grant SCHU 1503/4-2.

(C)2010 American Mathematical Society

Reverts to public domain 28 years from publication 2863 
mechanics, but it remains of considerable mathematical interest. It provides a natural measure of how well distributed points are on the surface of the sphere, and it also offers the possibility of characterizing important or beautiful configurations via extremal properties.

In most cases the optimal configuration depends on the potential function, but occasionally it does not. In [CK], Cohn and Kumar introduced the concept of universally optimal configurations, which minimize energy not only under all inversepower laws but also under the broader class of completely monotonic potential functions (as functions of squared Euclidean distance). In $\mathbb{R}^{2}$ the vertices of any regular polygon form a universally optimal configuration. The vertex sets of the regular tetrahedron, octahedron, or icosahedron are universally optimal, but there are no larger examples in $\mathbb{R}^{3}$. Higher-dimensional examples include the vertices of the regular simplex and cross polytope (or hyperoctahedron), and also various exceptional examples, notably the vertices of the regular 600 -cell, the $E_{8}$ root system, the Schläfli configuration of 27 points in $\mathbb{R}^{6}$ corresponding to the 27 lines on a cubic surface, and the minimal vectors of the Leech lattice. A number of the sporadic finite simple groups act on universal optima. See Tables 1 and 2 in [BBCGKS] for a list of the known and conjectured universal optima, as well as for a discussion of how many more there might be. (They appear to be quite rare.)

Every universal optimum is balanced (as we will explain below), but balanced configurations do not necessarily minimize energy even locally. In the space of configurations, balanced configurations are universal critical points for energy, but they are frequently saddle points. For example, the vertices of a cube are balanced, but one can lower the energy by rotating the vertices of a facet. Nevertheless, being balanced is an important necessary condition for universal optimality.

The simplest reason a configuration would be balanced is due to its symmetry: the net forces inherit this symmetry, which can constrain them to point orthogonally to the sphere. More precisely, call a finite subset $\mathcal{C} \subset S^{n-1}$ group-balanced if for every $x \in \mathcal{C}$, the stabilizer of $x$ in the isometry group of $\mathcal{C}$ fixes no vectors in $\mathbb{R}^{n}$ other than the multiples of $x$. A group-balanced configuration must be balanced, because the net force on $x$ is invariant under the stabilizer of $x$ and is thus orthogonal to the sphere.

In his 1957 paper $[\mathrm{L}$, Leech completely classified the balanced configurations in $S^{2}$. His classification shows that they are all group-balanced, and in fact the complete list can be derived easily from this assertion using the classification of finite subgroups of $O(3)$. However, Leech's proof is based on extensive case analysis, and it does not separate cleanly in this way. Furthermore, the techniques do not seem to apply to higher dimensions.

It is natural to wonder whether all balanced configurations are group-balanced in higher dimensions. If true, that could help explain the symmetry of the known universal optima. However, in this paper we show that balanced configurations need not be group-balanced. Among several counterexamples, we construct a configuration of 25 points in $\mathbb{R}^{12}$ that is balanced yet has no nontrivial symmetries.

This result is compatible with the general philosophy that it is difficult to find conditions that imply symmetry in high dimensions, short of simply imposing the symmetry by fiat. We prove that if a configuration is a sufficiently strong spherical design relative to the number of distances between points in it, then it is automatically balanced (see Theorem 2.1). Every spectral embedding of a strongly regular 
graph satisfies this condition (see Section 3). There exist strongly regular graphs with no nontrivial symmetries, and their spectral embeddings are balanced but not group-balanced.

Before we proceed to the proofs, it is useful to rephrase the condition of being balanced as follows: a configuration $\mathcal{C}$ is balanced if and only if for every $x \in \mathcal{C}$ and every real number $u$, the sum $S_{u}(x)$ of all $y \in \mathcal{C}$ whose inner product with $x$ is $u$ is a multiple of $x$. The reason is that the contribution to the net force on $x$ from the particles at a fixed distance is in the span of $x$ and $S_{u}(x)$. Since we are using arbitrary force laws, each contribution from a fixed distance must independently be orthogonal to the sphere (since we can weight them however we desire). Note that a group-balanced configuration $\mathcal{C}$ clearly satisfies this criterion: for every $x \in \mathcal{C}$ and every real number $u$, the sum $S_{u}(x)$ is itself fixed by the stabilizer of $x$ and hence must be a multiple of $x$.

An immediate consequence of this characterization of balanced configurations is that it is easy to check whether a given configuration is balanced. By contrast, it seems difficult to check whether a configuration is universally optimal. For example, the paper [BBCGKS] describes a 40-point configuration in $\mathbb{R}^{10}$ that appears to be universally optimal, but so far no proof is known.

So far we have not explained why universal optima must be balanced. Any optimal configuration must be in equilibrium under the force laws corresponding to the potential functions it minimizes, but no configuration could possibly minimize all potential functions simultaneously (universal optima minimize a large but still restricted class of potentials). The explanation is that a configuration is balanced if and only if it is balanced for merely the class of inverse-power force laws. In the latter case, we cannot weight the force contributions from different distances independently. However, as the exponent of the force law tends to infinity, the force contribution from the shortest distance will dominate unless it acts orthogonally to the sphere. This observation can be used to isolate each force contribution in order by distance. Alternatively, we can argue that the configuration is balanced under any linear combination of inverse-power laws and hence any polynomial in the reciprocal of distance. We can then isolate any single distance by choosing that polynomial to vanish at all the other distances.

\section{SPheriCAL DESIGNS}

Recall that a spherical t-design in $S^{n-1}$ is a (nonempty) finite subset $\mathcal{C}$ of $S^{n-1}$ such that for every polynomial $p: \mathbb{R}^{n} \rightarrow \mathbb{R}$ of total degree at most $t$, the average of $p$ over $\mathcal{C}$ equals its average over all of $S^{n-1}$. In other words,

$$
\frac{1}{|\mathcal{C}|} \sum_{x \in \mathcal{C}} p(x)=\frac{1}{\operatorname{vol}\left(S^{n-1}\right)} \int_{S^{n-1}} p(x) d \mu(x),
$$

where $\mu$ denotes the surface measure on $S^{n-1}$ and $\operatorname{vol}\left(S^{n-1}\right)$ is of course not the volume of the enclosed ball but rather $\int_{S^{n-1}} d \mu(x)$.

Theorem 2.1. Let $\mathcal{C} \subset S^{n-1}$ be a spherical t-design. If for each $x \in \mathcal{C}$,

$$
|\{\langle x, y\rangle: y \in \mathcal{C}, y \neq \pm x\}| \leq t,
$$

then $\mathcal{C}$ is balanced.

Here, $\langle\cdot, \cdot\rangle$ denotes the usual Euclidean inner product. 
Proof. Let $x$ be any element of $\mathcal{C}$, and let $u_{1}, \ldots, u_{k}$ be the inner products between $x$ and the elements of $\mathcal{C}$ other than $\pm x$. By assumption, $k \leq t$. We wish to show that for each $i$, the sum $S_{u_{i}}(x)$ of all $z \in \mathcal{C}$ such that $\langle z, x\rangle=u_{i}$ is a multiple of $x$.

Given any vector $y \in \mathbb{R}^{n}$ and integer $i$ satisfying $1 \leq i \leq k$, define the degree $k$ polynomial $p: \mathbb{R}^{n} \rightarrow \mathbb{R}$ by

$$
p(z)=\langle y, z\rangle \prod_{j: 1 \leq j \leq k, j \neq i}\left(\langle x, z\rangle-u_{j}\right) .
$$

Suppose now that $y$ is orthogonal to $x$. Then the average of $p$ over $S^{n-1}$ vanishes, because on the cross sections of the sphere on which $\langle x, z\rangle$ is constant, each factor $\langle x, z\rangle-u_{j}$ is constant, while $\langle y, z\rangle$ is an odd function on such a cross section. More precisely, under the map $z \mapsto 2\langle x, z\rangle x-z$ (which preserves the component of $z$ in the direction of $x$ and multiplies everything orthogonal to $x$ by -1 ), the inner product with $x$ is preserved while the inner product with $y$ is multiplied by -1 . Since $\mathcal{C}$ is a $t$-design, it follows that the sum of $p(z)$ over $z \in \mathcal{C}$ also vanishes.

Most of the terms in this sum vanish: when $z= \pm x$, we have $\langle y, z\rangle=0$, and when $\langle x, z\rangle=u_{j}$, the product vanishes unless $j=i$. It follows that the sum of $p(z)$ over $z \in \mathcal{C}$ equals

$$
\sum_{z \in \mathcal{C}:\langle z, x\rangle=u_{i}}\langle y, z\rangle \prod_{j: 1 \leq j \leq k, j \neq i}\left(u_{i}-u_{j}\right)=\left(\prod_{j: 1 \leq j \leq k, j \neq i}\left(u_{i}-u_{j}\right)\right)\left\langle y, S_{u_{i}}(x)\right\rangle .
$$

Because the first factor is nonzero, we conclude that $S_{u_{i}}(x)$ must be orthogonal to $y$. Because this holds for all $y$ orthogonal to $x$, it follows that $S_{u_{i}}(x)$ is a multiple of $x$, as desired.

Examples. The vertices of a cube form a spherical 3-design, and only two inner products other than \pm 1 occur between them, so Theorem 2.1 implies that the cube is balanced. On the other hand, not every group-balanced configuration satisfies the hypotheses of the theorem. For example, the configuration in $S^{2}$ formed by the north and south poles and a ring of $k$ equally spaced points around the equator is group-balanced, but it is not even a 2-design if $k \neq 4$. In Section 3 we will show that Theorem 2.1 applies to some configurations that are not group-balanced, so the two sufficient conditions for being balanced are incomparable.

\section{Counterexamples From Strongly Regular GraphS}

Every spectral embedding of a strongly regular graph is both a spherical 2-design and a 2-distance set, so by Theorem 2.1 they are all balanced. Recall that to form a spectral embedding of a strongly regular graph with $N$ vertices, one orthogonally projects the standard orthonormal basis of $\mathbb{R}^{N}$ to a nontrivial eigenspace of the adjacency matrix of the graph. See Sections 2 and 3 of [CGS] for a brief review of the theory of spectral embeddings. Theorem 4.2 in CGS gives the details of the result that every spectral embedding is a 2-design, a fact previously noted as part of Example 9.1 in DGS].

The symmetry group of such a configuration is simply the combinatorial automorphism group of the graph, so it suffices to find a strongly regular graph with no nontrivial automorphisms. According to Brouwer's tables [B1], the smallest such graph is a 25-vertex graph with parameters $(25,12,5,6)$ (the same as those of the Paley graph for the 25-element field), which has a spectral embedding in $\mathbb{R}^{12}$. See 


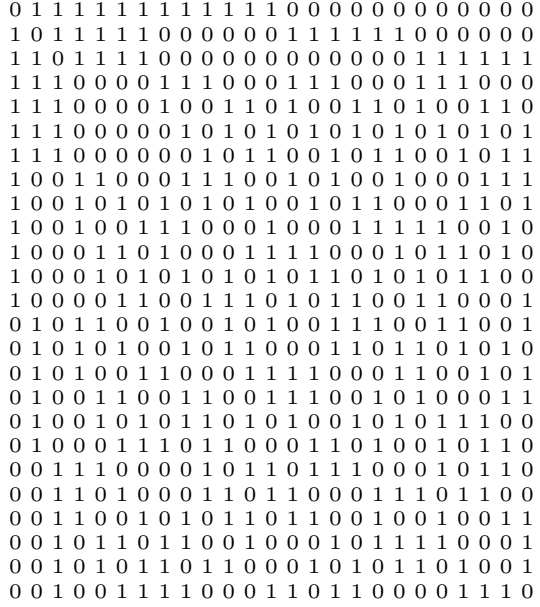

FiguRE 1. An adjacency matrix of a $(25,12,5,6)$ strongly regular graph with no nontrivial automorphisms.

Figure 1 for an adjacency matrix. Verifying that this graph has no automorphisms takes a moderate amount of calculation, which is best done by computer.

In fact, there are two such graphs with no nontrivial automorphisms (the other is the complement of the graph in Figure 1). Paulus classified the $(25,12,5,6)$ strongly regular graphs in $[\mathrm{P}$; unfortunately, his paper was never published. There are fifteen such graphs, whose automorphism groups have a variety of sizes: two have order 1 , four have order 2 , two have order 3 , four have order 6 , two have order 72 , and one has order 600 (the Paley graph). See B2 for more information.

The Paulus graphs give the lowest-dimensional balanced configurations we have found that have trivial symmetry groups. However, there are lower-dimensional counterexamples (with some symmetry but not enough to be group-balanced). The lowest-dimensional one we have constructed is in $\mathbb{R}^{7}$, and it can be built as follows; fortunately, no computer calculations are needed.

Let $\mathcal{C}_{n}$ consist of the $n(n+1) / 2$ midpoints of the edges of a regular simplex in $\mathbb{R}^{n}$ (scaled so that $\mathcal{C}_{n} \subset S^{n-1}$ ). This configuration is a 2 -distance set, with the distances corresponding to whether the associated edges of the simplex intersect or not. To compute the inner products, note that if $x_{1}, \ldots, x_{n+1}$ are the vertices of a regular simplex with $\left|x_{i}\right|^{2}=1$ for all $i$ (and hence $\left\langle x_{i}, x_{j}\right\rangle=-1 / n$ for $i \neq j$ ), then for $i \neq j$ and $k \neq \ell$,

$$
\left\langle x_{i}+x_{j}, x_{k}+x_{\ell}\right\rangle= \begin{cases}2-2 / n & \text { if }\{i, j\}=\{k, \ell\}, \\ 1-3 / n & \text { if }|\{i, j\} \cap\{k, \ell\}|=1, \text { and } \\ -4 / n & \text { if }\{i, j\} \cap\{k, \ell\}=\emptyset\end{cases}
$$

Thus, when we renormalize the vectors $x_{i}+x_{j}$ to lie on the unit sphere, we find that the inner products between them are $(1-3 / n) /(2-2 / n)=(n-3) /(2 n-2)$ and $-(4 / n) /(2-2 / n)=-2 /(n-1)$.

For $n>3$, the symmetry group of $\mathcal{C}_{n}$ is the same as that of the original simplex (namely the symmetric group on the vertices of the simplex). Clearly, that group acts on $\mathcal{C}_{n}$. To see that $\mathcal{C}_{n}$ has no other symmetries, we will show that the original 
simplex can be constructed from it in such a way as to be preserved by all symmetries of $\mathcal{C}_{n}$. Specifically, consider the subsets of $\mathcal{C}_{n}$ of size $n$ in which all pairs of points are at the minimal distance; the sums of these subsets are proportional to the vertices of the original simplex. To see why, note that such a subset corresponds to a collection of $n$ pairwise-intersecting edges of the original simplex. They must be exactly the edges containing one of the vertices of the simplex: once two intersecting edges are specified, only one other edge can intersect both without containing their common vertex, so at most three edges can intersect pairwise without containing a common vertex. (Note that this conclusion genuinely requires that $n>3$, because $\mathcal{C}_{3}$ is an octahedron, which has more symmetry than the tetrahedron from which it was derived.)

When $n=7$ the inner products in $\mathcal{C}_{7}$ are simply $\pm 1 / 3$. The coincidence that these inner products are negatives of each other is deeper than it appears, and it plays a role in several useful constructions. For example, the union of $\mathcal{C}_{7}$ and its antipode $-\mathcal{C}_{7}$ is a 3 -distance set, while in other dimensions it would be a 5 -distance set. In fact, $\mathcal{C}_{7} \cup\left(-\mathcal{C}_{7}\right)$ is the unique 56-point universal optimum in $\mathbb{R}^{7}$, and it is invariant under the Weyl group of $E_{7}$. We will make use of the unusual inner products in $\mathcal{C}_{7}$ to construct a modification of it that is balanced but not groupbalanced.

Within $\mathcal{C}_{7}$, there are regular tetrahedra (i.e., quadruples of points with all inner products $-1 / 3)$. Geometrically, such a tetrahedron corresponds to a set of four disjoint edges in the original simplex, and there is a unique such set up to symmetry, since the simplex in $\mathbb{R}^{7}$ has eight vertices and all permutations of these vertices are symmetries. Choose a set of four disjoint edges and call them the distinguished edges.

We now define a modified configuration $\mathcal{C}_{7}^{\prime}$ by replacing each point in this tetrahedron by its antipode. Replacing the regular tetrahedron preserves the 2-design property, because the tetrahedron is itself a 2-design within the 2 -sphere it spans. In particular, for every polynomial of total degree at most 2 , its sum over the original tetrahedron is the same as its sum over the antipodal tetrahedron. Furthermore, when we replace the tetrahedron, all inner products remain $\pm 1 / 3$ (some are simply multiplied by -1 ). Thus, the resulting configuration $\mathcal{C}_{7}^{\prime}$ remains both a 2-distance set and a 2-design, so it is balanced by Theorem 2.1 .

However, the process of inverting a tetrahedron reduces the symmetry group.

Lemma 3.1. The configuration $\mathcal{C}_{7}^{\prime}$ has only $4 ! \cdot 2^{4}=384$ symmetries, namely the permutations of the vertices of that original simplex that preserve the set of four distinguished edges.

Proof. There are clearly $4 ! \cdot 2^{4}$ symmetries of $\mathcal{C}_{7}$ that preserve the set of distinguished edges of the simplex (they can be permuted arbitrarily and their endpoints can be swapped). All of these symmetries preserve $\mathcal{C}_{7}^{\prime}$.

To show that there are no further symmetries, it suffices to show that the distinguished tetrahedron in $\mathcal{C}_{7}^{\prime}$ is preserved under all symmetries. (For then the antipodal tetrahedron is also preserved, and hence $\mathcal{C}_{7}$ is preserved as well.) Label the vertices of the original simplex $1,2, \ldots, 8$, and suppose that the distinguished edges correspond to the pairs $12,34,56$, and 78 . Label the points of $\mathcal{C}_{7}^{\prime}$ by the pairs for the corresponding edges.

There are at most two orbits under the symmetry group of $\mathcal{C}_{7}^{\prime}$, one containing $12,34,56$, and 78 and the other containing the remaining points. We wish to show 
that these sets do not in fact form a single orbit. To separate the two orbits, we will count the number of regular tetrahedra each point is contained in. (We drop the word "regular" below.) The answer will be seven for the four distinguished points and eleven for the other points, so they cannot lie in the same orbit.

Before beginning, we need a criterion for when the inner product between two points in $\mathcal{C}_{7}^{\prime}$ is $-1 / 3$. If both points are distinguished or both are nondistinguished, then that occurs exactly when their label pairs are disjoint. If one point is distinguished and the other is not, then it occurs exactly when their label pairs intersect.

Now it is straightforward to count the tetrahedra containing a distinguished point; without loss of generality, call the point 12 . There is one tetrahedron of distinguished points, namely $\{12,34,56,78\}$. If we include a second distinguished point, say 34 , then there are two ways to complete the tetrahedron using two nondistinguished points, namely $\{12,34,13,24\}$ and $\{12,34,14,23\}$ (the two additional pairs must be disjoint and each intersect both 12 and 34). Because there are three choices for the second distinguished point, this yields six tetrahedra. Finally, it is impossible to form a tetrahedron using point 12 and three nondistinguished points (one cannot choose three disjoint pairs that each intersect 12). Thus, 12 is contained in seven tetrahedra.

To complete the proof, we need only show that a nondistinguished point, without loss of generality 13, is contained in more than seven tetrahedra. There is a unique tetrahedron containing point 13 and two distinguished points, namely $\{13,12,34,24\}$. (There are only two distinguished points that overlap with 13, namely 12 and 34; then the fourth point 24 is determined.) No tetrahedron can contain a single distinguished point, as we saw in the previous paragraph, and if a tetrahedron contains three distinguished points, then it must contain the fourth. Thus, the only remaining possibility is that all the points are nondistinguished. The three other points in the tetrahedron must be labeled with disjoint pairs from $\{2,4,5,6,7,8\}$, and the labels 56 and 78 are not allowed (because those points are distinguished). There are $6 ! /\left(2 !^{3} \cdot 3 !\right)=15$ ways to split $\{2,4,5,6,7,8\}$ into three disjoint pairs. Among them, three contain the pair 56, three contain the pair 78 , and one contains both pairs. Thus, there are $15-3-3+1=10$ possibilities containing neither 56 nor 78 . In total, the point 13 is contained in eleven tetrahedra. Since it is contained in more than seven tetrahedra, we see that points 12 and 13 are in different orbits, as desired.

By Lemma 3.1, there are two orbits of points in $\mathcal{C}_{7}^{\prime}$, namely the four points in the tetrahedron and the remaining 24 points. The stabilizer of any point in the large orbit actually fixes two such points. Specifically, consider the edge in the original simplex that corresponds to the point. It shares its vertices with two of the four distinguished edges (each vertex is in a unique distinguished edge), and there is another edge that connects the other two vertices of those distinguished edges. For example, in the notation of the proof of Lemma 3.1 the edge 13 has the companion 24. This second edge has the same stabilizer as the first. It follows that $\mathcal{C}_{7}^{\prime}$ is not group-balanced.

If we interpret the configuration $\mathcal{C}_{7}^{\prime}$ as a graph by using its shorter distance to define edges, then we get a strongly regular graph, with parameters $(28,12,6,4)$, the same as those of $\mathcal{C}_{7}$. In fact, every 2-design 2-distance set yields a strongly regular graph by Theorem 7.4 of [DGS]. We have checked using Brouwer's list [B1] that spectral embeddings of strongly regular graphs do not yield counterexamples 
in lower dimensions. It suffices to consider graphs with at most 27 vertices, since by Theorem 4.8 in [DGS] no two-distance set in $S^{5}$ contains more than 27 points. Aside from the degenerate case of complete multipartite graphs and their complements, the full list of strongly regular graphs with spectral embeddings in six or fewer dimensions is the pentagon, the Paley graph on 9 vertices, the Petersen graph, the Paley graph on 13 vertices, the line graph of $K_{6}$, the Clebsch graph, the Shrikhande graph, the $4 \times 4$ lattice graph, the line graph of $K_{7}$, the Schläfli graph, and the complements of these graphs. It is straightforward to check that these graphs all have group-balanced spectral embeddings. Of course there may be low-dimensional counterexamples of other forms.

We suspect that there are no counterexamples in $\mathbb{R}^{4}$ :

Conjecture 3.2. In $\mathbb{R}^{4}$, every balanced configuration is group-balanced.

If true, this conjecture would lead to a complete classification of balanced configurations in $\mathbb{R}^{4}$, because all the finite subgroups of $O(4)$ are known (see for example CSm $)$. It is likely that, using such a classification, one could prove completeness for the list of known universal optima in $\mathbb{R}^{4}$, namely the regular simplices, cross polytope, and 600-cell, but we have not completed this calculation.

In $\mathbb{R}^{5}$ or $\mathbb{R}^{6}$, we are not willing to hazard a guess as to whether all balanced configurations are group-balanced. The construction of $\mathcal{C}_{7}^{\prime}$ uses such an ad hoc approach that it provides little guidance about lower dimensions.

\section{Counterexamples from lattices}

In higher dimensions, we can use lattices to construct counterexamples that do not arise from strongly regular graphs. For example, consider the lattice $\Lambda\left(G_{2}\right)$ in the Koch-Venkov list of extremal even unimodular lattices in $\mathbb{R}^{32}$ (see $\mathrm{KV}$, p. 212] or the Nebe-Sloane catalogue [NS] of lattices). This lattice has 146880 minimal vectors. When they are renormalized to be unit vectors, only five inner products occur besides \pm 1 (namely, $\pm 1 / 2, \pm 1 / 4$, and 0 ). By Corollary 3.1 of [BV], this configuration is a spherical 7-design. Hence, by Theorem 2.1 it is balanced. However, $\operatorname{Aut}\left(\Lambda\left(G_{2}\right)\right)$ is a relatively small group, of order $3 \cdot 2^{12}$, and one can check by computer calculations that some minimal vectors have trivial stabilizers. (The lattice is generated by its minimal vectors, and thus it and its kissing configuration have the same symmetry group.) The kissing configuration of $\Lambda\left(G_{2}\right)$ is therefore balanced but not group-balanced.

The case of $\Lambda\left(G_{2}\right)$ is particularly simple since some stabilizers are trivial, but one can also construct lower-dimensional counterexamples using lattices. For example, let $L$ be the unique 2-modular lattice in dimension 20 with Gram matrix determinant $2^{10}$, minimal norm 4 , and automorphism group $2 \cdot M_{12} \cdot 2$ (see $\mathrm{BV}$, p. 101] or [NS). The kissing number of $L$ is 3960, and its automorphism group (which is, as above, the same as the symmetry group of its kissing configuration) acts transitively on the minimal vectors. The kissing configuration is a spherical 5-design (again by Corollary 3.1 in [BV]), and only five distances occur between distinct, nonantipodal points. Thus, by Theorem 2.1 the kissing configuration of $L$ is balanced. However, computer calculations show that the stabilizer of a point fixes a 2-dimensional subspace of $\mathbb{R}^{20}$, and thus the configuration is not group-balanced.

The kissing configurations of the extremal even unimodular lattices $P_{48 p}$ and $P_{48 q}$ in $\mathbb{R}^{48}$ (see [CSl, p. 149]) are also balanced but not group-balanced. They have 
52416000 minimal vectors, with inner products $\pm 1, \pm 1 / 2, \pm 1 / 3, \pm 1 / 6$, and 0 after rescaling to the unit sphere. By Corollary 3.1 in [BV], the kissing configurations are spherical 11-designs, so by Theorem 2.1 they are balanced. However, in both cases there are points with trivial stabilizers, so they are not group-balanced. Checking this is more computationally intensive than in the previous two cases. Fortunately, for the bases listed in NS, in both cases the first basis vector is a minimal vector with trivial stabilizer, and this triviality is easily established by simply enumerating the entire orbit. (The automorphism groups of $P_{48 p}$ and $P_{48 q}$ have orders 72864 and 103776, respectively.) We expect that the same holds for every extremal even unimodular lattice in $\mathbb{R}^{48}$, but they have not been fully classified, and we do not see how to prove it except for checking each case individually.

\section{Euclidean Balanced CONFigurations}

The concept of a balanced configuration generalizes naturally to Euclidean space: a discrete subset $\mathcal{C} \subset \mathbb{R}^{n}$ is balanced if for every $x \in \mathcal{C}$ and every distance $d$, the set $\{y \in \mathcal{C}:|x-y|=d\}$ either is empty or has centroid $x$. As in the spherical case, this characterization is equivalent to being in equilibrium under all pairwise forces that vanish past some radius (to avoid convergence issues).

The concept of a group-balanced configuration generalizes as well. Let $\operatorname{Aut}(\mathcal{C})$ denote the set of rigid motions of $\mathbb{R}^{n}$ preserving $\mathcal{C}$. Then $\mathcal{C}$ is group-balanced if for every $x \in \mathcal{C}$, the stabilizer of $x$ in $\operatorname{Aut}(\mathcal{C})$ fixes only the point $x$. For example, every lattice in Euclidean space is group-balanced, because the stabilizer of each lattice point contains the operation of reflection through that point. Clearly, groupbalanced configurations are balanced, because the centroid of $\{y \in \mathcal{C}:|x-y|=d\}$ is fixed by the stabilizer of $x$.

Conjecture 5.1. Every balanced discrete subset of $\mathbb{R}^{2}$ is group-balanced.

Conjecture 5.1 can likely be proved using ideas similar to those used by Leech in $\mathrm{L}$ to prove the analogue for $S^{2}$, but we have not completed a proof.

Conjecture 5.2. If $n$ is sufficiently large, then there exists a discrete subset of $\mathbb{R}^{n}$ that is balanced but not group-balanced.

One might hope to prove Conjecture 5.2 using an analogue of Theorem 2.1 . Although we have not succeeded with this approach, one can indeed generalize several of the ingredients to Euclidean space: the analogue of a polynomial is a radial function from $\mathbb{R}^{n}$ to $\mathbb{R}$ whose Fourier transform has compact support (i.e., the function is an entire function of exponential type), and the analogue of the degree of the polynomial is the radius of the support. Instead of having a bounded number of roots, such a function has a bounded density of roots. The notion of a spherical design also generalizes to Euclidean space as follows. A configuration $\mathcal{C}$ with density 1 (i.e., one point per unit volume in space) is a "Euclidean $r$-design" if whenever $f$ is a radial Schwartz function with $\operatorname{supp}(\widehat{f}) \subseteq B_{r}(0)$, the average of $\sum_{y \in \mathcal{C}} f(x-y)$ over $x \in \mathcal{C}$ equals $\int f(x-y) d y=\widehat{f}(0)$. (The average or even the sum may not make sense if $\mathcal{C}$ is pathological, but for example they are always well-defined for periodic configurations.) It is plausible that an analogue of Theorem 2.1 is true in the Euclidean setting, but we have not attempted to state or prove a precise analogue, because it is not clear that it would have any interesting applications. 


\section{ACKNOWLEDGEMEnTs}

We thank Richard Green and the anonymous referee for their suggestions and feedback.

\section{REFERENCES}

[BV] C. Bachoc and B. Venkov, Modular forms, lattices and spherical designs, Réseaux euclidiens, designs sphériques et formes modulaires, 87-111, Monogr. Enseign. Math. 37, Enseignement Math., Geneva, 2001. MR1878746 (2003d:11096)

[BBCGKS] B. Ballinger, G. Blekherman, H. Cohn, N. Giansiracusa, E. Kelly, and A. Schürmann, Experimental study of energy-minimizing point configurations on spheres, Experiment. Math. 18 (2009), 257-283.

[B1] A. E. Brouwer, Parameters of strongly regular graphs, tables published electronically at http://www.win.tue.nl/ ${ }^{\sim}$ aeb/graphs/srg/srgtab.html.

[B2] A. E. Brouwer, Paulus graphs, tables published electronically at http://www.win. tue.nl/ aeb/graphs/Paulus.html

[CGS] P. J. Cameron, J. M. Goethals, and J. J. Seidel, Strongly regular graphs having strongly regular subconstituents, J. Algebra 55 (1978), 257-280. MR523457(81d:05034)

[CK] H. Cohn and A. Kumar, Universally optimal distribution of points on spheres, J. Amer. Math. Soc. 20 (2007), 99-148. MR2257398 (2007h:52009)

[CSl] J. Conway and N. J. A. Sloane, Sphere Packings, Lattices and Groups, third edition, Grundlehren der Mathematischen Wissenschaften, 290, Springer-Verlag, New York, 1999. MR:1662447 (2000b:11077)

[CSm] J. H. Conway and D. A. Smith, On Quaternions and Octonions: Their Geometry, Arithmetic, and Symmetry. A K Peters, Ltd., Natick, MA, 2003. MR.1957212 (2004a:17002)

[DGS] P. Delsarte, J. Goethals, and J. Seidel, Spherical codes and designs, Geometriae Dedicata 6 (1977), 363-388. MR0485471 (58:5302)

[KV] H. Koch and B. Venkov, Über gerade unimodulare Gitter der Dimension 32. III, Math. Nachr. 152 (1991), 191-213. MR.1121234 (92j:11064)

[L] J. Leech, Equilibrium of sets of particles on a sphere, Math. Gaz. 41 (1957), 81-90. MR $0086325(19: 165 b)$

[NS] G. Nebe and N. Sloane, A catalogue of lattices, tables published electronically at http://www.research.att.com/ njas/lattices/

[P] A. J. L. Paulus, Conference matrices and graphs of order 26, Technische Hogeschool Eindhoven, report WSK 73/06, Eindhoven, 1973, 89 pp.

[T] J. J. Thomson, On the structure of the atom: An investigation of the stability and periods of oscillation of a number of corpuscles arranged at equal intervals around the circumference of a circle; with application of the results to the theory of atomic structure, Phil. Mag. (6) 7 (1904), 237-265. 02142

Microsoft Research New England, One Memorial Drive, Cambridge, Massachusetts

E-mail address: cohn@microsoft.com

Department of Mathematics, Harvard University, Cambridge, Massachusetts 02138

E-mail address: elkies@math.harvard.edu

Department of Mathematics, Massachusetts Institute of Technology, Cambridge, MASSACHUSETTS 02139

E-mail address: abhinav@math.mit.edu

Institute of Applied Mathematics, TU Delft, Mekelweg 4, 2628 CD Delft, The NETHERLANDS

E-mail address: a.schurmann@tudelft.nl 